\title{
Trastuzumab and docetaxel in a preclinical organotypic breast cancer model using tissue slices from mammary fat pad: Translational relevance
}

\author{
LOREDANA VESCI $^{1 *}$, VALERIA CAROLLO ${ }^{3 *}$, GIUSEPPE ROSCILLI $^{2}$, LUIGI AURISICCHIO $^{2}$, \\ FABIANA FOSCA FERRARA ${ }^{2}$, LUIGI SPAGNOLI ${ }^{3}$ and RITA DE SANTIS ${ }^{1}$
}

${ }^{1}$ Biotechnology, Research and Development, Sigma-Tau Industrie Farmaceutiche Riunite S.p.A., I-00040 Pomezia;
${ }^{2}$ Takis Biotechnology, Castel Romano, I-00128 Rome; ${ }^{3}$ Tissue Macro Array Lab, University of Tor Vergata, I-00133 Rome, Italy

Received April 20, 2015; Accepted May 15, 2015

DOI: 10.3892/or.2015.4074

\begin{abstract}
With the ever-increasing number of drugs approved to treat cancers, selection of the optimal treatment regimen for an individual patient is challenging. Breast cancer complexity requires novel predictive methods and tools. In the present study, we set up experimental conditions to obtain an 'ex vivo' organotypic culture from xenotransplanted mice aiming at recapitulating the human clinical condition. The effect of trastuzumab (large biological molecule) and docetaxel (small chemical entity) was subsequently investigated on this organotypic model and compared with in vivo and in vitro activity on tumor cells. Tissue slices of $200 \mu \mathrm{m}$ were obtained from mammary fat pad of SCID mice xenotransplanted with human MCF-7 breast cancer cells. Viability and proliferation were evaluated by 3-(4,5-dimethylthiazol-2-yl)-2,5-diphenyltetrazolium bromide (MTT) colorimetric assay and Ki-67 immunohistochemistry,and apoptosis by cleaved caspase-3 immunohistochemistry. In vivo antitumor activity of trastuzumab and docetaxel was determined by caliper measurement of tumor volume and Ki-67 expression on explanted masses by immunohistochemistry. A Teflon support and normoxia were necessary experimental conditions to obtain high viability of excised breast cancer infiltrated mammary fat pad slices upon $48 \mathrm{~h}$ cultivation, as shown by MTT proliferation assay, and Ki-67 expression. Breast cancer tissue slices treated for $48 \mathrm{~h}$ with trastuzumab or docetaxel showed a significant dose-dependent reduction of viability by MTT assay. Consistently, both drugs down-modulated Ki-67 and increased cleaved caspase-3. Tumor masses collected from docetaxel- or trastuzumab-treated mice showed a similar
\end{abstract}

Correspondence to: Dr Loredana Vesci, Biotechnology, Research and Development, Sigma-Tau Industrie Farmaceutiche Riunite S.p.A., via Pontina Km. 30.400, I-00040 Pomezia, Rome, Italy

E-mail: loredana.vesci@sigma-tau.it

${ }^{*}$ Contributed equally

Key words: tissue slices, breast cancer, mammary fat pad, trastuzumab, docetaxel reduction of proliferation markers. By contrast, MCF-7 cell cultures were significantly inhibited by docetaxel but not by trastuzumab. Tumor tissue slices represent a more predictive experimental cancer model compared to cell cultures for both small and large molecule antitumor efficacy. This observation supports the relevance of microenvironment in the overall tumor biology and response to therapeutics.

\section{Introduction}

Breast cancer is the most common type of cancer in the world with more than 1.3 million patients and a mortality rate of $\sim 450,000$ deaths/year (1). The high incidence of disease is suggestive of slow progress in the prevention programs. In the treatment of women with localized disease, mortality rates have been improved, but the median survival time in the metastatic setting is only 25 months (2). Systemic chemotherapy as treatment of choice for most cancer types and its relatively modest improvement in survival associated with significant toxicity, highlighted the need over the past decade to develop targeted therapies. Targeted drugs can restore the deregulated signalling transduction pathway in which a mutated gene and its encoded protein is involved (3). Targeted therapy represents the major hope against cancer and a substantial step towards personalized medicine, since this type of drugs is mostly active in cancer cells without affecting normal healthy cells. However, the efficacy of these single signal transduction pathway inhibitors is in most cases modest, thus the progress against cancer is slow and it is translated into a few weeks of survival prolongation. Elucidation of the mechanisms beyond intrinsic and acquired tumor resistance to therapies is hampered by high inter-patient and intratumor heterogeneity (4-7). Consequently, a patient-specific experimental approach is needed to analyze individual responses to therapies. Based on comprehensive gene expression profiling, breast tumors are classified into at least three major subtypes: luminal, human epidermal growth factor receptor $2^{+}\left(\right.$HER2 $\left.^{+}\right)$ and basal-like $(8,9)$, with different risk factors for incidence, response to treatment, disease progression and preferential organ sites of metastases (10-12). Antitumor drugs, presently used in the clinical practice, have been previously characterized 
in the two-dimensional in vitro culture and in vivo xenograft tumor models. A broad analysis on investigational drugs, done at the National Cancer Institute, pointed out at a poor correlation between preclinical data from in vitro models and tumor xenografts and phase II efficacy data leading to the conclusion that only compounds that are successful in a large number of different models are likely to be effective in the clinic (13). Thus, frequent failures in drug development can be explained by the fact that the existing preclinical models do not represent the complexity (heterogeneity) that is typical of human tumors. We have, therefore, recently explored tissue slice technology via the Krumdieck tissue slicer, by preserving the tissues in the three-dimensional structure, it allows the setting up of a powerful and representative ex vivo tumor model. Moreover, taking into account the notion that cancer cell lines, passaged in vitro for years, may not reflect the biology of in vivo tumors, we compared the activity of docetaxel (small molecule) and trastuzumab (large molecule), both commonly used for breast cancer therapy, in cancer cell lines and organotypic tissue slices.

\section{Materials and methods}

Materials. The primary antibodies used in the present study were monoclonal rabbit anti-human Ki-67 antigen (NB600-1252; Novus Biologicals, Littleton, CO, USA) and cleaved caspase-3 (\#9664; Cell Signaling Technology, Danvers, MA, USA). The secondary antibody was biotinylated goat anti-rabbit (\#E0432; Dako, Denmark).

Ethics statement. All studies were performed in accordance with the 'Directive 2010/63/UE' on the protection of animals used for scientific purposes, made effective in Italy by the Legislative Decree 4 March 2014, n. 26, and applying the principles of $3 R$ s (i.e., to replace, reduce and refine). Mice were purchased from Harlan Laboratories (Udine, Italy). All procedures performed on the animals were approved by the Animal Welfare Body and authorized by the Italian Ministry of Health, 46/2014-PR. At the end of the treatment period and before necropsy, mice were euthanized by compressed $\mathrm{CO}_{2}$ gas in a cylinder as indicated in the American Veterinary Medical Association (AVMA) Panel on Euthanasia according to the 1998 UKCCCR Guidelines for the Welfare of Animals in Experimental Neoplasia.

Tumor xenograft in mammary fat pad. MCF-7 breast tumor cells were injected into the abdominal fat pad of SCID Beige ( $7 \times 10^{6}$ cells $/ 100 \mu \mathrm{l} /$ mouse), $24 \mathrm{~h}$ after the subcutaneous implantation of estrogen pellets containing $17 \beta$-estradiol (0.72 mg/pellet, 60-day release/mouse) (\#SE-121; Innovative Research of America, Sarasota, FL, USA). Tumor lesions were measured with a Vernier caliper twice a week to reach a volume of around $300 \mathrm{~mm}^{3}$ prior to collection for tissue slice preparation.

For in vivo drug efficacy evaluation, tumor-bearing mice were also treated with docetaxel (Sigma-Aldrich, St. Louis, MO, USA) at $15 \mathrm{mg} / 10 \mathrm{ml} / \mathrm{kg}$ i.p. (q7dx3, once a week for 3 weeks) and trastuzumab at $10 \mathrm{mg} / 10 \mathrm{ml} / \mathrm{kg}$ i.p. (q7dx3) (Herceptin; Roche S.p.A., Milan, Italy). Tumor lesions were measured with a Vernier caliper twice a week to reach a volume of around $300 \mathrm{~mm}^{3}$ before to be collected for analysis of marker proliferation.

Tumor explantation. Mice were sacrificed, tumors explanted and immediately cut using the Vibratome VT1200 (Leica, Germany) to obtain $200 \mu \mathrm{M}$ thick slices of the whole tumors.

Tissue slice maintaining conditions. To set the best experimental conditions, tumor slices were maintained in floating normoxic conditions for a maximum of 4 days using a modular incubator chamber $37^{\circ} \mathrm{C}$ in 6-multiwell plates, using Dulbecco's modified Eagle's medium (DMEM) with $10 \%$ FBS, a mix of penicillin-streptomycin and $1 \%$ L-glutamine as culture medium. Medium change was performed every $24 \mathrm{~h}$.

Subsequently, Teflon supporting and normoxia were evaluated. Tumor slices were maintained at $37^{\circ} \mathrm{C}$ and $5 \% \mathrm{CO}_{2}$ (normoxic conditions) for a maximum of 4 days on organotypic Teflon inserts (\#PICM01250 MilliCell; Millipore, Billerica, MA, USA) in 6-multiwell plates, using DMEM (\#D5921; Sigma-Aldrich) with $10 \%$ FBS, $1 \%$ L-glutamine, a mix of penicillin-streptomycin as culture medium. Medium change was performed every $24 \mathrm{~h}$.

Cell viability determination in tissue slices. The viability of tumor slices was evaluated using the 3-(4,5-dimethylthiazol-2-yl)-2,5-diphenyltetrazolium bromide (MTT) (\#M5655; Sigma-Aldrich) under normoxia $\left(21 \% \mathrm{O}_{2}\right)$ after a maximum of 3 days of incubation. Moreover, the integrity of the slices was assessed by hematoxylin and eosin (H\&E) staining.

Immunohistochemistry. Immunochemistry analysis was performed by employing paraffin sections. Tissue slices of $200 \mu \mathrm{m}$ were paraffin-embedded (horizontal orientation). The sections were incubated with primary antibodies overnight. After washing, secondary antibodies were applied at 1:200 dilutions for $30 \mathrm{~min}$. Images were acquired using a Nikon microscope (Eclipse 80i, Nikon, Japan) with a Nikon digital camera (DXM1200F). H\&E staining was performed to examine the extent of the slice integrity.

Drug treatment of slice cultures. Slices obtained from three mice were evaluated in triplicate for MTT assay 2 or 3 days after slicing. For treatment of slices, docetaxel (Sigma-Aldrich) and trastuzumab (Herceptin) were used. The concentrations were chosen on the basis of results obtained from tumor cell growth inhibition assay. Before testing docetaxel in its final concentrations of 200-20 nM, it was dissolved in dimethyl sulfoxide (DMSO) and diluted in culture medium. Trastuzumab was tested at the concentrations of $20-0.02 \mu \mathrm{g} / \mathrm{ml}$. The incubation period for treatment was up to 2 days. At the end of the treatment, tissue slices were incubated with $5 \mathrm{mg} / \mathrm{ml}$ of MTT at $37^{\circ} \mathrm{C}$ for $1 \mathrm{~h}$, harvested and precipitated-salt extracted by incubation with $0.1 \mathrm{M} \mathrm{HCl}$-isopropyl alcohol at room temperature for $40 \mathrm{~min}$. Viability values were determined by dividing the optical density of the formazan at $570 \mathrm{~nm}$ by the dry weight of the explants.

Growth inhibition tumor cell assay. MTT assay was performed for evaluation of the cell viability within 3 days of culture. Tumor cells were seeded 3,000 cells/well. Upon 24 h, tumor 
A
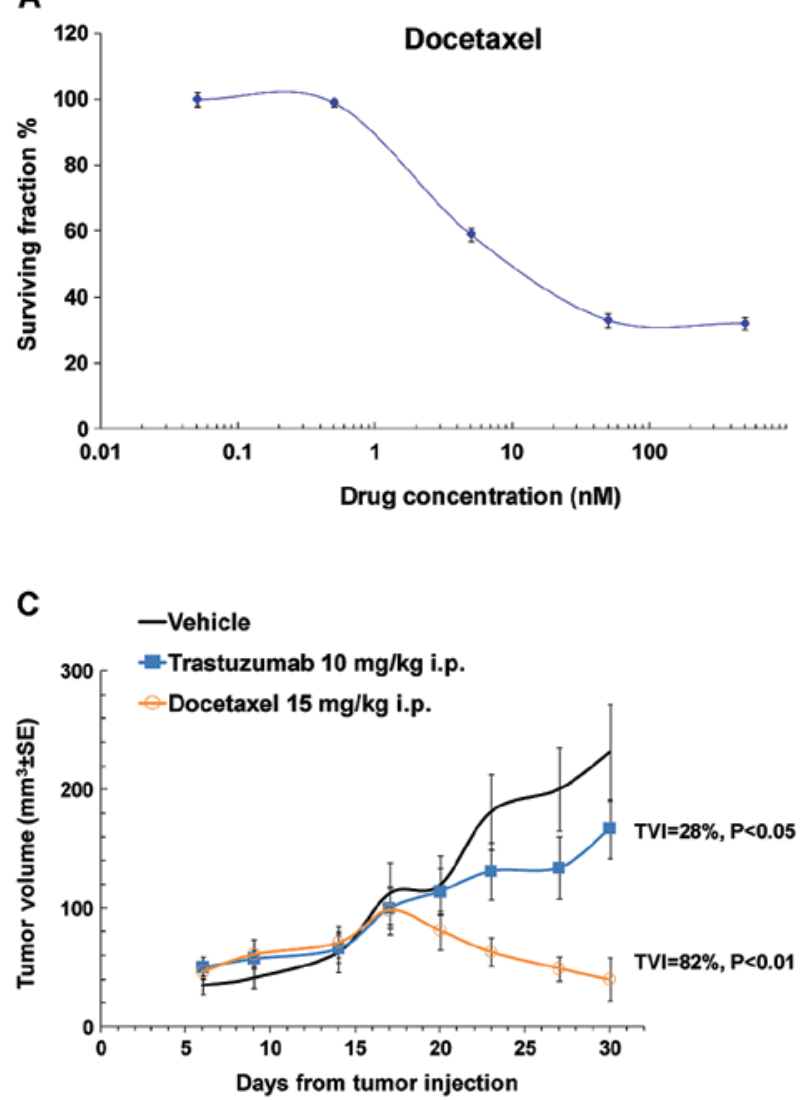

B
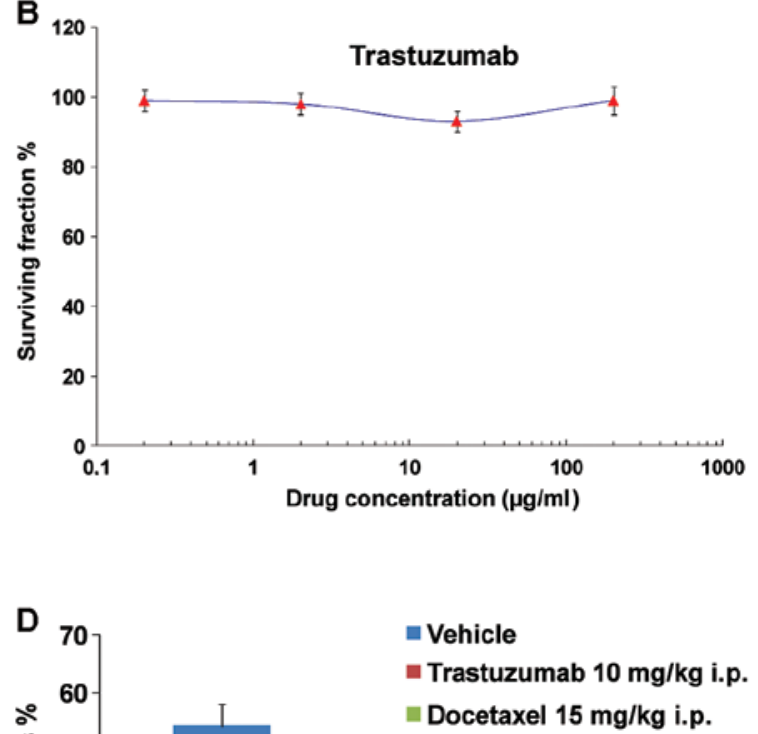

Docetaxel $15 \mathrm{mg} / \mathrm{kg}$ i.p.

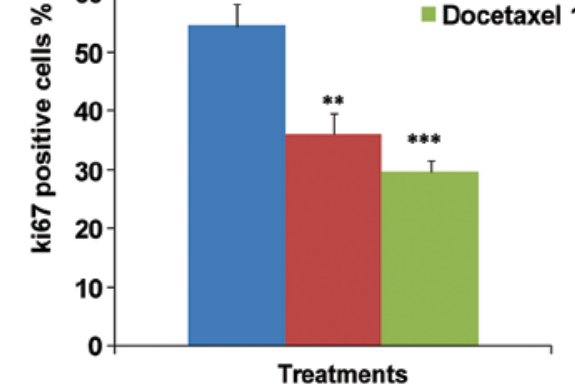

Figure 1. In vitro and in vivo antitumor activity of docetaxel and trastuzumab on MCF-7 breast carcinoma. (A and B) In vitro MCF-7 tumor cell growth inhibition induced by docetaxel and trastuzumab evaluated after 3 days of culture. Cell survival was evaluated by MTT assay. Data are means of two experiments and each point was in triplicate. The $\mathrm{IC}_{50}$ values expressed as mean $\pm \mathrm{SE}$ were $7 \pm 0.05 \mathrm{nM}$ for docetaxel and $>200 \mu \mathrm{g} / \mathrm{ml}$ for trastuzumab. $(\mathrm{C}) \mathrm{MCF}-7$ mammary fat pad tumor-bearing mice were treated as indicated according to the schedule q7dx3 ( $=8 / \mathrm{group}$ ), starting 6 days after tumor implantation. TVI was evaluated 10 days after the last treatment. The statistical comparison of drug-treated group vs. the vehicle treated group was performed by Mann-Whitney $\mathrm{U}$ test. (D) Percent of $\mathrm{Ki}-67^{+}$cells, as detected by immunohistochemical analysis in mammary fat pad tumor lesions of mice treated as indicated ( 8 mice/group) $\left({ }^{* *} \mathrm{P}<0.01\right.$ and ${ }^{* * *} \mathrm{P}<0.001$ vs. vehicle-treated group, Mann-Whitney $\mathrm{U}$ test).

cells were treated with different concentrations of trastuzumab (200-20 $\mu \mathrm{g} / \mathrm{ml})$ and docetaxel (500-0.5 nM) for 3 days. At the end of treatment, the medium was discarded, cells were washed twice with PBS, and replaced by MTT-containing medium. The plates were incubated at $37^{\circ} \mathrm{C}$ for $4 \mathrm{~h}$. Then the MTT solution was discarded and without washing, DMSO was added to dissolve the formazan formed. After $15 \mathrm{~min}$ incubation, cell plates were transferred to the microplate reader (Victor, Wallac) and the absorbance at $570 \mathrm{~nm}$ was measured. The results were expressed as percent of drug-treated viable cells in comparison with vehicle-treated cells. The $\mathrm{IC}_{50}$ values were calculated using four-parameter fit by ALLFIT program.

Statistical analysis. All data are expressed as the mean value \pm standard error (SE) and differences between groups were analyzed using Mann-Whitney U test. Mean values are considered significantly different at $\mathrm{P}<0.05$.

\section{Results}

In vitro and in vivo antitumor activity of docetaxel and trastuzumab. In order to evaluate the in vitro and in vivo antitumor activity of docetaxel and trastuzumab, standard human MCF-7 cell culture and a tumor xenograft study in nude mice were carried out. These tumor cells were first of all analysed for ERBB2 membrane expression by FACS analysis with $97.6 \%$ of tumor cells positive for ERBB2-conjugated antibody (R\&S, FAB 11299) (data not shown). Tumor cell proliferation was tested by MTT assay after 3 days of culture showing a dose-dependent inhibitory effect of docetaxel but not trastuzumab, resulting in an $\mathrm{IC}_{50}$ of $7 \pm 0.1 \mathrm{nM}$ and $>200 \mu \mathrm{g} / \mathrm{ml}$, respectively (Fig. 1A and B). Consistently with in vitro data, docetaxel strongly inhibited the growth of the MCF-7 xenografts (TVI=82\%; $\mathrm{P}<0.01)$ whereas trastuzumab exhibited a lower but significant antitumor activity (TVI=28\%; $\mathrm{P}<0.05$ ) (Fig. 1C). Reduction of Ki-67 expression correlated with both docetaxel-dependent and trastuzumab-dependent antitumor activity (Fig. 1D). Ki-67 is an important marker predicting recurrence, prognosis and overall survival in breast cancer patients (14-16). It has also been associated with positive axillary lymph nodes in most studies $(17,18)$. Recently, Ki-67 was integrated as a prognostic factor into molecular typing in prognosis of patients with luminal B breast cancer (19). Therefore, we investigated Ki-67 expression in further tumor slice experiments.

Tumor slice preparation. The set up of ex vivo organotypic cultures of human breast carcinoma was carried out before 
A

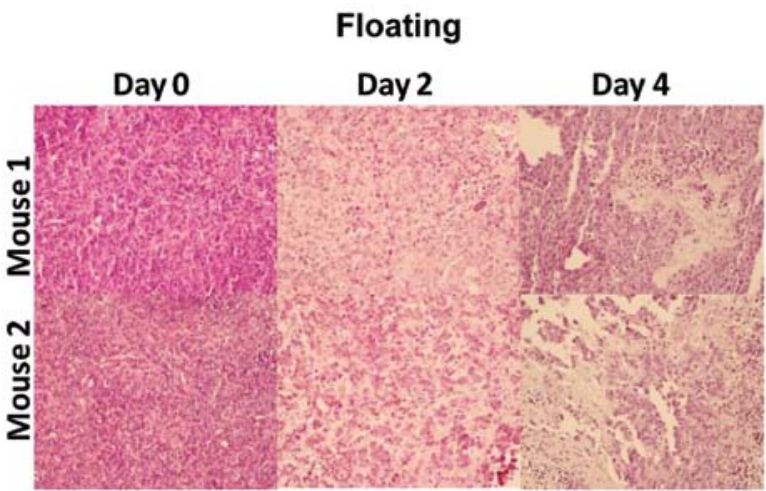

B

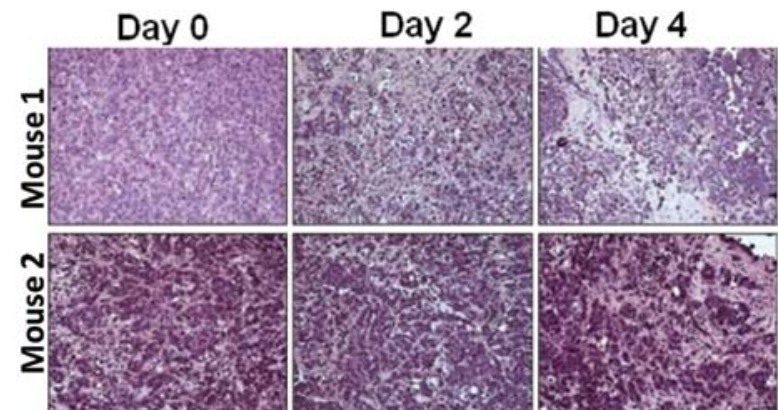

$\mathrm{C}_{100}$

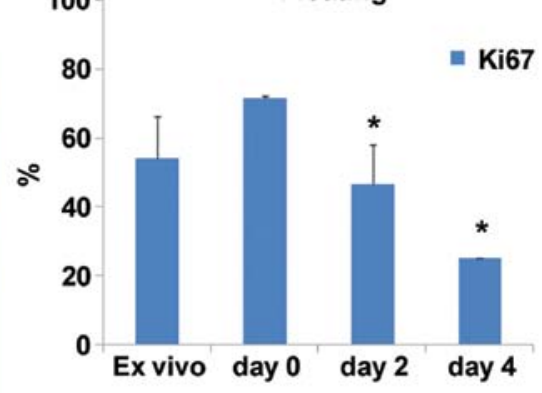

D

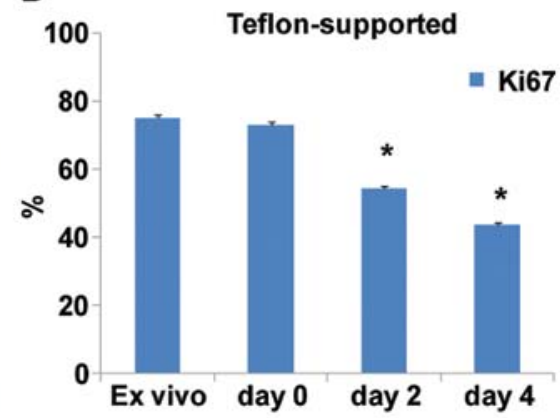

Figure 2. Tissue integrity of tissue slices in floating and teflon supported conditions. (A and B) Tissue slices were processed immediately or kept for 2 or 4 days in culture medium, floating or Teflon-supported conditions, then fixed in formalin and paraffin-embedded for analysis of H\&E staining. Images were from two representative mice and were digitally scanned x20 (AlexaSoft X-PRO). (C and D) Percentage of Ki-67 as detected by immunohistochemical analysis in mammary fat pad of tumor implanted samples (ex vivo) and tissue slices after 0,2 or 4 days of culture in floating or Teflon-supported conditions. The data are means from 3 mice in triplicate ( ${ }^{*} \mathrm{P}<0.05$ vs. tissue slice on day 0 , Mann-Whitney U test). H\&E, hetoxylin and eosin.

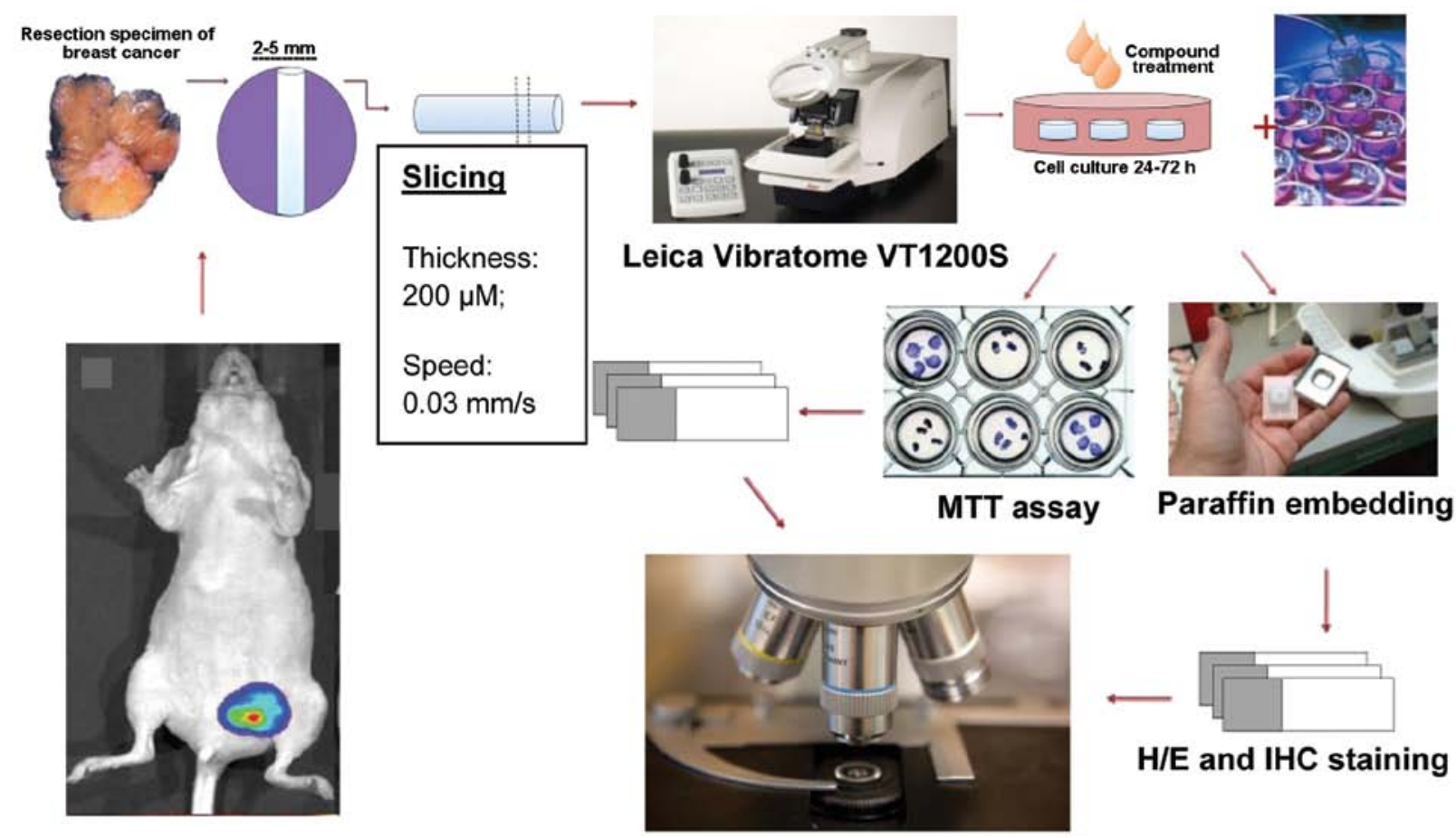

Figure 3. Representative scheme of tissue slice production and analysis.

drug testing. Tumor slices of $200 \mu \mathrm{m}$ were obtained from MCF-7 xenotransplanted mammary fat pads by the use of a vibratome. Slices were subjected to histological analysis to identify the best experimental conditions to preserve the tissue culture viability. The slices were maintened in normoxia for 2 or 4 days in floating or in Teflon-supported conditions before analyses. H\&E staining of tumor slices revealed a high qualitative viability in the Teflon-supported compared to floating 
A
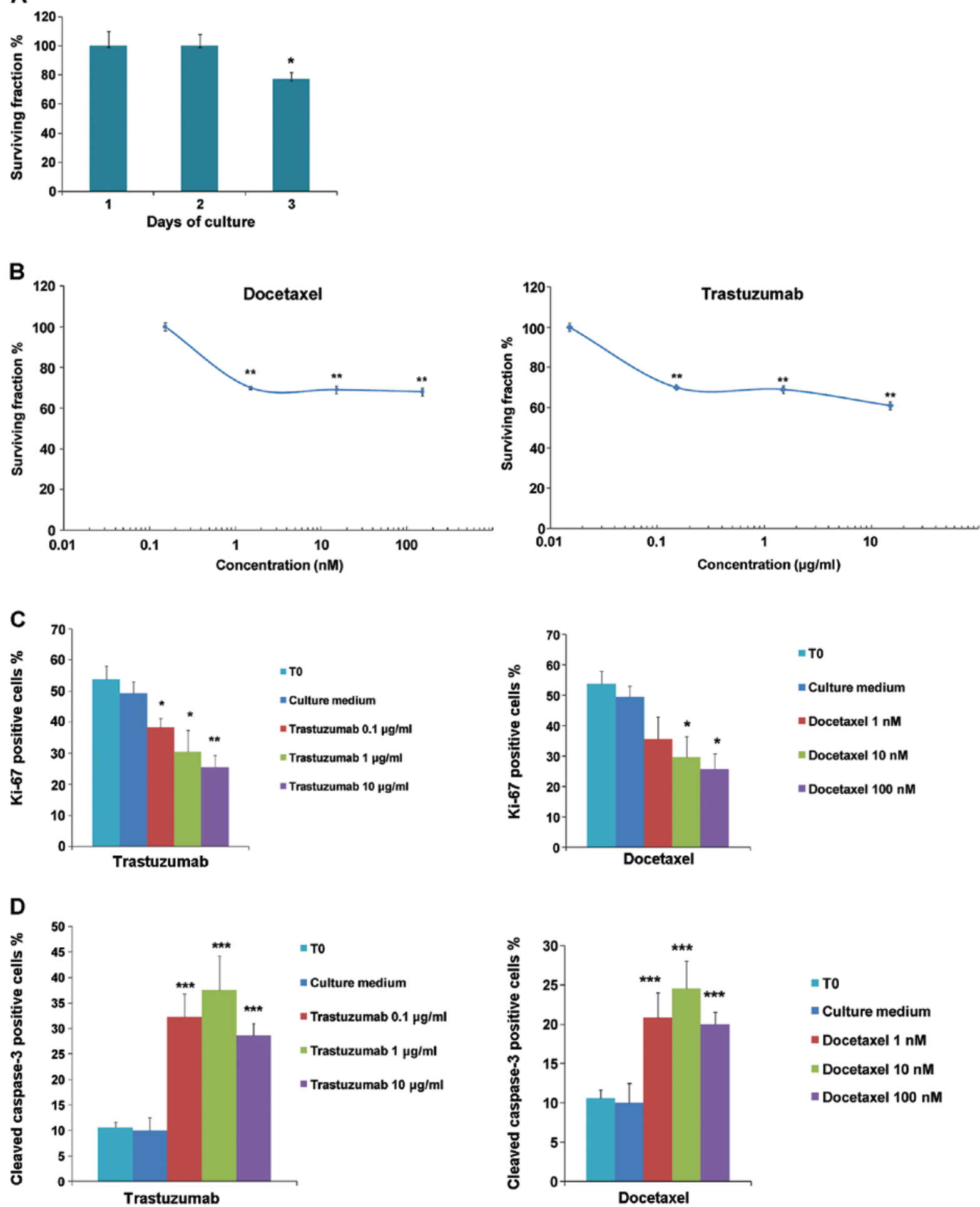

Figure 4. Viability of tissue slices on Teflon support upon docetaxel or trastuzumab treatment. (A) MTT assay was carried out on tissue slices after 1-3 days in culture. Data are mean $\pm \mathrm{SE}$ in triplicate of 3 slices/time-point. ( $\mathrm{P}<0.05$ vs. day $1 \mathrm{Mann}-$ Whitney $\mathrm{U}$ test). (B) Teflon-supported tissue slices treated with trastuzumab or docetaxel for 2 days. Each point is the mean \pm SE of triplicate slices ( ${ }^{* *} \mathrm{P}<0.01$ vs. culture medium, Mann-Whitney $\mathrm{U}$ test). (C and D) Percentage of Ki-67 and cleaved caspase- 3 positive cells of tissue slices processed immediately $(\mathrm{t} 0)$ or kept 2 days in culture medium with or without indicated drugs. Data were mean $\pm \mathrm{SE}$ from two tissue slices untreated and drug-treated $\left({ }^{*} \mathrm{P}<0.05,{ }^{* *} \mathrm{P}<0.01\right.$ and ${ }^{* * *} \mathrm{P}<0.001$ vs. tissue slice on day 0 , Mann-Whitney $\mathrm{U}$ test).

condition (Fig. 2A and B). Accordingly, significantly decreased expression of Ki-67 was found by immunohistochemistry in floating slices compared to Teflon-supported slices (Fig. 2C and D).

These results indicated that an inert support is useful to keep slices viable and tumor cells in a proliferative status, thus indicating that tissue slices represent a physiologically more relevant model compared to tissue cultures to study solid cancer. Moreover, despite the lack of blood supply the viability of Teflon-supported $200 \mu \mathrm{m}$ thick tissue slices, maintained in normoxia, suggests an adequate nutrient diffusion from the culture medium. 

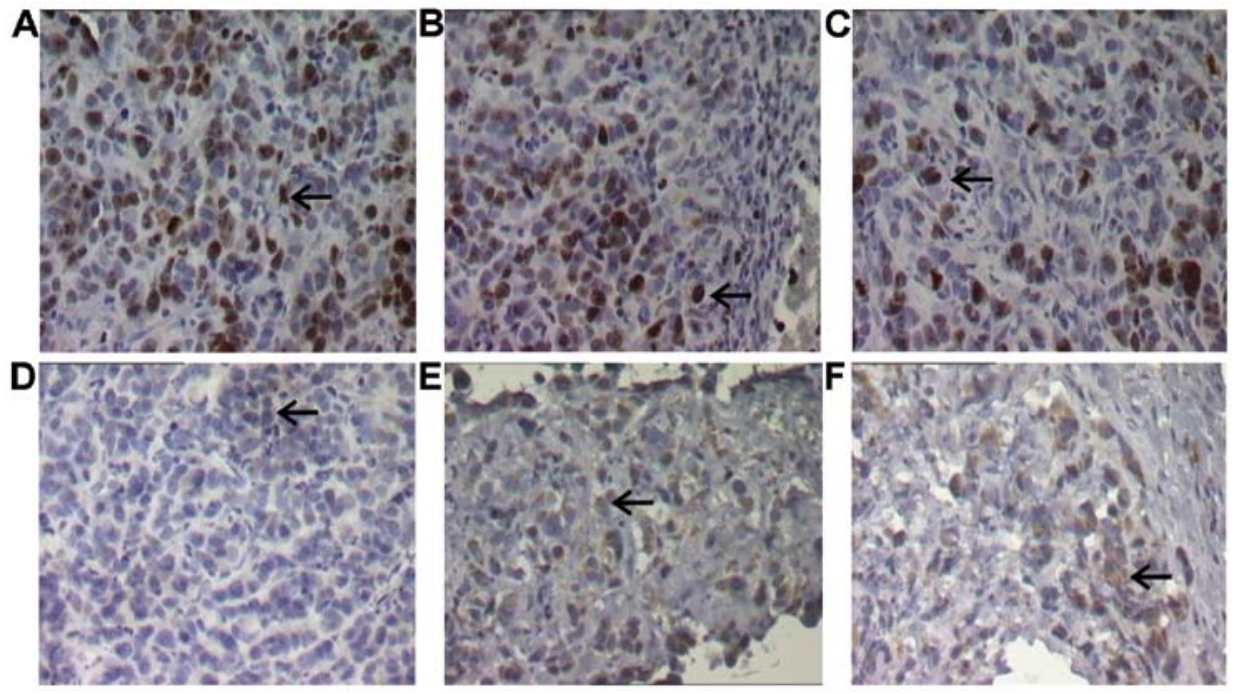

Figure 5. Immunohistochemistry of tissue slices treated with trastuzumab and docetaxel. (Ki-67, arrows; A-C) and (cleaved caspase-3, arrows; D-F) were quantified by counting the number of positively stained cells in 4 fields of view at $\mathrm{x} 40$. Images are representative of untreated (A, D), trastuzumab at $100 \mu \mathrm{g} /$ $\mathrm{ml}(\mathrm{B}$ and $\mathrm{E})$ and docetaxel at $100 \mathrm{nM}(\mathrm{C}$ and F). Images were digitally scanned x40 (AlexaSoft X-PRO).

A schematic representation of the procedure to obtain organotypic breast cancer tissue slices from MCF-7 mammary fat pad xenotransplant is shown in Fig. 3.

Evaluation of docetaxel and trastuzumab antitumor activity on breast cancer tissue slices. Because substantial morphological integrity of tissues, defined as preservation of general architecture including epithelial structures and their spatial relationship to stroma, was observed in tumor tissue slices up to 2 days of culture (Fig. 2). MTT assay was performed to quantify viability. Data in Fig. 4A show similar number of viable cells after 1 or 2 days dropping to $\sim 80 \%$ after 3 days of cultivation. Further experiments were then performed cultivating the tissue slices for 2 days in the presence of different concentrations of docetaxel or trastuzumab. MTT assay revealed a significant dose-dependent loss of viable cells upon treatment with both drugs (Mann-Whitney U test; ${ }^{* *} \mathrm{P}<0.01$ ) (Fig. 4B). In agreement with this result, the percentage of proliferating cells assessed by Ki-67 immunostaining significantly decreased upon docetaxel or trastuzumab exposure (Fig. 4C), paralleled by increased percentage of cleaved caspase- 3 positive cells (Fig. 4D). In particular, biomarkers of proliferation and apoptosis used to quantify effects of trastuzumab and docetaxel were evaluated by counting numbers of positively stained cells (Fig. 5A-F). Taken together, these data show that tissue slices can better reflect the in vivo experimental outcome.

\section{Discussion}

In the present study we report a method to obtain organotypic breast cancer tissue slices useful to evaluate the efficacy of both small and a large molecule drugs. The advantage of the tissue slice cultures is that the complex cross-talk between matrix and cells as well as the cellular heterogeneity and susceptibility to drugs is preserved avoiding potential source of artefacts deriving from isolation of tumor cells from their biological environment $(20,21)$. Moreover, the presence of
ECM provides important differentiation cues, for example, by signalling via Toll-like receptors and integrins (22-24), thus further supporting the use of tissue slice method for screening of therapeutics. Organotypic tissue slice cultures offer a more attractive option compared to cell line cultures or subcutaneous xenografts both lacking the complexity of a tumor nested in a mammary gland (25-27). Tissue slice samples with a thickness between $400-800 \mu \mathrm{m}$, cultured $24 \mathrm{~h}$ were previously used to evaluate the transcriptional effects of $1,25(\mathrm{OH})_{2} \mathrm{D}_{3}$ in breast cancer benefitting from the heterogeneous combination of epithelial and stromal cells that secrete a variety factors affecting the overall response of tumor cells to the vitamin (28). Tissue slices of $250 \mu \mathrm{m}$ from human primary invasive ductal breast tumors were also employed to test tamoxifen and doxorubicin activity, confirming the complexity and heterogeneity of breast cancer among patients (29). Primary tissue slices of $400 \mu \mathrm{m}$ were cultured in the presence of rapamycin, showing that such a culture method preserves the tumor AKT/mTOR pathway activity (30). Moreover, slice cultures of $350 \mu \mathrm{m}$ from head and neck squamous cell carcinoma from patients were also used to test cisplatin, docetaxel and cetuximab allowing the design of personalized therapies and investigation of mechanisms of tumor resistance $(31,32)$. It is becoming increasingly evident that the development of cancer and the response to anti-cancer drugs not only depend on genetic alterations but also on specific interactions between tumor cells and surrounding tissue components. In invasive breast carcinoma, differentiated myoepithelial cells and intact basement membranes are lost and tumor cells are in direct contact with an activated collagenous tumor stroma (27). To simulate such conditions either three-dimensional tissue cultures using several biomatrices or co-culture experiments with tumor fibroblasts have been performed. However, these systems cannot mimic the complex tissue architecture and the high degree of variability seen in individual tumors. Therefore, to evaluate the activity of docetaxel and trastuzumab antitumor drugs we used tissue slice cultures starting from orthotopic human breast cancer xenografts in mice and compared results 
with in vivo efficacy. Our data support the use of the patient's tissue slices as predictive translational model for personalized therapies.

So far, neither in vitro nor ex vivo testing technologies have gained a significant impact in predicting clinical efficacy of therapeutic treatments. Present data can be framed within a widespread effort to identify testing conditions useful to increase the success rate of drug investigation, thus facilitating translational medicine.

Here we provide evidence that breast cancer tissue slice is a more useful model than cell cultures to predict antitumor efficacy of both small and large molecules. Sensitivity of breast tumors to anticancer drugs depends upon dynamic interactions between epithelial tumor cells and their microenvironment including stromal cells and extracellular matrix. Moreover, in addition to cancer cell viability, this model improves the understanding of the impact of a given treatment on stromal and endothelial cells and the distribution of a biologic, a small molecule or viral vectors within tumor microenvironment. Another potential application may include the ex vivo addition of immune cells for OncoImmunology studies.

In conclusion, tissue slices represent an optimal tool to investigate therapeutics and further studies to evaluate this model for novel investigational drugs are warranted.

\section{References}

1. Ferlay J, Shin HR, Bray F, Forman D, Mathers C and Parkin DM: Estimates of worldwide burden of cancer in 2008: GLOBOCAN 2008. Int J Cancer 127: 2893-2917, 2010.

2. Siegel R, Naishadham D and Jemal A: Cancer statistics, 2013. CA Cancer J Clin 63: 11-30, 2013.

3. Rask-Andersen M, Almén MS and Schiöth HB: Trends in the exploitation of novel drug targets. Nat Rev Drug Discov 10: 579-590, 2011.

4. Klein CA: Selection and adaptation during metastatic cancer progression. Nature 501: 365-372, 2013.

5. Bedard PL, Hansen AR, Ratain MJ and Siu LL: Tumour heterogeneity in the clinic. Nature 501: 355-364, 2013.

6. Roukos DH: Beyond HER2 and trastuzumab: Heterogeneity, systems biology, and cancer origin research may guide the future for personalized treatment of very early but aggressive breast cancer. J Clin Oncol 28: e279-e280, author reply e282-e283, 2010.

7. Curtis C, Shah SP, Chin SF, Turashvili G, Rueda OM,Dunning MJ, Speed D, Lynch AG, Samarajiwa S, Yuan Y, et al; METABRIC Group: The genomic and transcriptomic architecture of 2,000 breast tumours reveals novel subgroups. Nature 486: 346-352, 2012.

8. Sørlie T, Perou CM, Tibshirani R, Aas T, Geisler S, Johnsen H, Hastie T, Eisen MB, van de Rijn M, Jeffrey SS, et al: Gene expression patterns of breast carcinomas distinguish tumor subclasses with clinical implications. Proc Natl Acad Sci USA 98: 10869-10874, 2001.

9. Perou CM, Sørlie T, Eisen MB, van de Rijn M, Jeffrey SS, Rees CA, Pollack JR, Ross DT, Johnsen H, Akslen LA, et al: Molecular portraits of human breast tumours. Nature 406: 747-752, 2000

10. Lehmann BD, Bauer JA, Chen X, Sanders ME, Chakravarthy AB, Shyr Y and Pietenpol JA: Identification of human triple-negative breast cancer subtypes and preclinical models for selection of targeted therapies. J Clin Invest 121: 2750-2767, 2011.

11. Prat A, Parker JS, Karginova O, Fan C, Livasy C, Herschkowitz JI, $\mathrm{He} \mathrm{X}$ and Perou CM: Phenotypic and molecular characterization of the claudin-low intrinsic subtype of breast cancer. Breast Cancer Res 12: R68, 2010.

12. Russnes HG, Vollan HK, Lingjaerde OC, Krasnitz A, Lundin P, Naume B, Sørlie T, Borgen E, Rye IH, Langerød A, et al: Genomic architecture characterizes tumor progression paths and fate in breast cancer patients. Sci Transl Med 2: 38-47, 2010.
13. Johnson JI, Decker S, Zaharevitz D, Rubinstein LV, Venditti JM, Schepartz S, Kalyandrug S, Christian M, Arbuck S, Hollingshead M, et al: Relationships between drug activity in NCI preclinical in vitro and in vivo models and early clinical trials. Br J Cancer 84: 1424-1431, 2001.

14. de Azambuja E, Cardoso F, de Castro G Jr, Colozza M, Mano MS, Durbecq V, Sotiriou C, Larsimont D, Piccart-Gebhart MJ and Paesmans M: Ki-67 as prognostic marker in early breast cancer: A meta-analysis of published studies involving 12,155 patients. Br J Cancer 96: 1504-1513, 2007.

15. Li FY, Wu SG, Zhou J, Sun JY, Lin Q, Lin HX, Guan XX and He ZY: Prognostic value of Ki-67 in breast cancer patients with positive axillary lymph nodes: A retrospective cohort study. PLoS One 9: e87264, 2014.

16. Stuart-Harris R, Caldas C, Pinder SE and Pharoah P: Proliferation markers and survival in early breast cancer: A systematic review and meta-analysis of 85 studies in 32,825 patients. Breast 17: 323-334, 2008

17. Urruticoechea A, Smith IE and Dowsett M: Proliferation marker Ki-67 in early breast cancer. J Clin Oncol 23: 7212-7220, 2005.

18. Jalava P, Kuopio T, Juntti-Patinen L, Kotkansalo T, Kronqvist P and Collan Y: Ki67 immunohistochemistry: a valuable marker in prognostication but with a risk of misclassification: proliferation subgroups formed based on Ki67 immunoreactivity and standardized mitotic index. Histopathology 48: 674-682, 2006.

19. Cheang MC, Chia SK, Voduc D, Gao D, Leung S, Snider J, Watson M, Davies S, Bernard PS, Parker JS, et al: Ki67 index, HER 2 status, and prognosis of patients with luminal B breast cancer. J Natl Cancer Inst 101: 736-750, 2009.

20. Pietras K and Ostman A: Hallmarks of cancer: Interactions with the tumor stroma. Exp Cell Res 316: 1324-1331, 2010.

21. Hanahan D and Weinberg RA: Hallmarks of cancer: The next generation. Cell 144: 646-674, 2011.

22. Moreth K, Brodbeck R, Babelova A, Gretz N, Spieker T, Zeng-Brouwers J, Pfeilschifter J, Young MF, Schaefer RM and Schaefer L: The proteoglycan biglycan regulates expression of the B cell chemoattractant CXCL13 and aggravates murine lupus nephritis. J Clin Invest 120: 4251-4272, 2010

23. Merline R, Moreth K, Beckmann J, Nastase MV, Zeng-Brouwers J, Tralhão JG, Lemarchand P, Pfeilschifter J, Schaefer RM, Iozzo RV, et al: Signaling by the matrix proteoglycan decorin controls inflammation and cancer through PDCD4 and MicroRNA-21. Sci Signal 4: ra75, 2011.

24. Alghisi GC, Ponsonnet L and Rüegg C: The integrin antagonist cilengitide activates alphaVbeta3, disrupts VE-cadherin localization at cell junctions and enhances permeability in endothelial cells. PLoS One 4: e4449, 2009.

25. Krumdieck CL, dos Santos JE and Ho KJ: A new instrument for the rapid preparation of tissue slices. Anal Biochem 104: 118-123, 1980.

26. Hood CJ and Parham DM: A simple method of tumour culture. Pathol Res Pract 194: 177-181, 1998.

27. van der Kuip H, Mürdter TE, Sonnenberg M, McClellan M, Gutzeit S, Gerteis A, Simon W, Fritz P, Aulitzky WE and Aulitzky WE: Short term culture of breast cancer tissues to study the activity of the anticancer drug taxol in an intact tumor environment. BMC Cancer 6: 86-92, 2006.

28. Milani C, Katayama ML, de Lyra EC, Welsh J, Campos LT, Brentani MM, Maciel MS, Roela RA, del Valle PR, Góes JC, et al: Transcriptional effects of 1,25 dihydroxyvitamin $\mathrm{D}(3)$ physiological and supra-physiological concentrations in breast cancer organotypic culture. BMC Cancer 13: 119, 2013.

29. Holliday DL, Moss MA, Pollock S, Lane S, Shaaban AM, Millican-Slater R, Nash C, Hanby AM and Speirs V: The practicalities of using tissue slices as preclinical organotypic breast cancer models. J Clin Pathol 66: 253-255, 2013.

30. Grosso SH, Katayama ML, Roela RA, Nonogaki S, Soares FA, Brentani H, Lima L, Folgueira MA, Waitzberg AF, Pasini FS, et al: Breast cancer tissue slices as a model for evaluation of response to rapamycin. Cell Tissue Res 352: 671-684, 2013.

31. Gerlach MM, Merz F, Wichmann G, Kubick C, Wittekind C, Lordick F, Dietz A, Bechmann I and Waitzberg AF: Slice cultures from head and neck squamous cell carcinoma: A novel test system for drug susceptibility and mechanisms of resistance. Br J Cancer 110: 479-488, 2014.

32. Goldhirsch A, Ingle JN, Gelber RD, Coates AS, Thürlimann B and Senn HJ; Panel members: Thresholds for therapies: Highlights of the St Gallen International Expert Consensus on the primary therapy of early breast cancer 2009. Ann Oncol 20: $1319-1329,2009$. 